1
BU--124-MA

February, 1996

Effects of treatment and prevalence-dependent recruitment on the dynamics of a fatal disease

Jorge X. Velasco-Hernández

Departamento de Matemáticas UAM-Iztapalapa

and Biometrics Unit, Cornell University.

Fred Brauer

Department of Mathematics, University of Wisconsin

Carlos Castillo-Chavez

Biometrics Unit, Cornell University

Running title: Prevalence-dependent recruitment.

Please send correspondence to the first author to:

324 Warren Hall

Biometrics Unit, Cornell University

Ithaca, NY. 14853-7801

Tel: $607-255-5488$

Fax: 607-255-4698

E-mail: jxv1@cornell.edu 
Effects of treatment and prevalence-dependent recruitment on the dynamics of a fatal disease

Jorge X. Velasco-Hernández

Departamento de Matemáticas UAM-Iztapalapa and Biometrics Unit, Cornell University.

Fred Brauer

Department of Mathematics, University of Wisconsin

Carlos Castillo-Chavez

Biometrics Unit, Cornell University

Running title: Prevalence-dependent recruitment.

Please send correspondence to the first author to:

324 Warren Hall

Biometrics Unit, Cornell University

Ithaca, NY. 14853-7801

Tel: 607-255-5488

Fax: 607-255-4698

E-mail: jxv1@cornell.edu 


\title{
Effects of treatment and prevalence-dependent recruitment on the dynamics of a fatal disease
}

\author{
Jorge X.Velasco-Hernández \\ Departamento de Matemáticas, UAM-Iztapalapa \\ and Biometric Unit Cornell University \\ Fred Brauer \\ Department of Mathematics, University of Wisconsin \\ Carlos Castillo-Chavez \\ Biometrics Unit, Cornell University
}

\begin{abstract}
This paper studies models for the sexual transmission of HIV/AIDS that incorporate changes in behavior as well as the effects associated with HIV treatment. The recruitment rate into the core is assumed to be a function of the prevalence of the disease within the core and it may trigger the existence of periodic solutions through Hopf bifurcations, provided that there is at least a weak demographic interaction with the non-core. The recruitment function is set up for two cases: dependence on the total proportion of infectious individuals and dependence only on the proportion of treated infectious individuals. In the general model, numerical evidence suggests that both cases may produce periodic solutions when the perception of risk of joining the core group is sufficiently high. Two limiting cases are also studied: when the growth rate of the core and non core groups are essentially the same, and when treatment has no effect on the transmission rate of infected individuals.
\end{abstract}

\section{Introduction}

Sexually-transmitted diseases are usually driven by a relatively small proportion of the sexually-active individuals. Hethcote and Yorke (1984) used the concept of core-group as a key element of disease management. Their work was motivated in part by their efforts to develop cost-effective methods 
for reducing the incidence of gonorrhea. They found out that disease management strategies aimed at the core group were the most effective. Their 'moving' endemic equilibrium provided epidemiologists and public health officials with a quantitative model of their effectiveness.

Drastic changes in behavior have been observed in some homosexuallyactive populations as a result of the effects of the AIDS epidemic in these communities (see Baldwin and Baldwin 1988, Curran et al. 1988, Fineberg 1988, Evans et al. 1989, Martin 1987, Saltzman et al. 1987, Shechter et al. 1988, van Griensven et al. 1989a,b, Wilkenstein 1988, McKusick et al. 1985, Shilts 1987, and Wiktor et al. 1990). The effects of these documented changes in behavior on HIV incidence, and the development of partially-effective drug treatments such as AZT, makes it even more difficult to forecast the timing and magnitude of this epidemic. Changes in behavior may influence disease dynamics in various ways including in the recruitment of new susceptibles, the level of sexual activity, the type of sexual practices, and the rate of partnership exchange. Treatment may affect epidemiological parameters by increasing the length of the infectious period while possibly reducing the infectivity per sexual contact.

In this work we explore the interaction among behavioral changes, treatment of HIV-infected patients, and the long-term dynamics of HIV using models for homosexually-active populations. We combine the approaches of two earlier types of models. The first class of models makes recruitment into a population a function of disease levels (Blythe et al. 1992, Brauer et al. 1992, 1993, Hadeler and Castillo-Chavez 1994) while the second incorporates treatment into HIV epidemiological models (Velasco-Hernández and Hsieh 1994; Hsieh and Velasco-Hernández 1994). This paper is organized as follows: Section 2 modifies an earlier model of Velasco-Hernández and Hsieh (1994); Section 3 introduces the main model, finds the equilibria, and computes the basic reproductive number; Section 4 deals with the stability analysis and in Section 5 we give the conclusions derived from our results.

\section{Prevalence-dependent recruitment: basic model}

We start by revisiting the model of Velasco-Hernández and Hsieh (1994). Let $S, U$, and $I$ denote the numbers of susceptible, untreated infectious, and treated infectious individuals respectively. If we denote by $B$ the per- 
susceptible incidence rate, then the model equations are:

$$
\begin{aligned}
\frac{d S}{d t} & =g(S, U, I)-B S-\mu S \\
\frac{d U}{d t} & =B S-(\mu+\nu) U-\sigma \frac{U}{N} \\
\frac{d I}{d t} & =\sigma \frac{U}{N}-\left(\mu+\nu^{\prime}\right) I
\end{aligned}
$$

where $g(S, U, I)$ is the recruitment rate into the population, $\mu^{-1}$ is the average length of the sexually-active life of an individual, $\sigma$ is the treatment rate, and $\nu^{-1}$ and $\left(\nu^{\prime}\right)^{-1}$ denote the lengths of the infective period for untreated and treated individuals respectively. The per-susceptible incidence rate is given by

$$
B=\frac{c \beta_{0} U+c^{\prime} \beta_{1} I}{N}
$$

where $N=S+U+I, c$ and $c^{\prime}$ are the average number of partners per unit time for members of treated and untreated infectious classes, respectively; $\beta_{0}$ and $\beta_{1}$ are the corresponding infectivity rates so that $c \beta_{0}$ and $c^{\prime} \beta_{1}$ are the net disease transmission rates of the treated and untreated infective class, respectively.

Brauer et al. (1992) studied a model without treated class and illustrated their results using the recruitment function $\Lambda \exp (-\kappa I / N)$. They showed the existence of stable limit cycle solutions analytically and numerically. Velasco-Hernández and Hsieh (1994) while assuming a constant recruitment rate introduced the dynamics of the treated class.

The goal of this article is to study the role of both factors (the existence of a treated class and non-constant recruitment) on the transmission dynamics of HIV.

In addition, in this article we illustrate our results numerically and analytically using the specific recruitment function

$$
g(S, U, I)=\Lambda e^{-\kappa \frac{v U+w I}{N}}
$$

where the constant $\kappa$ is an index of the strength that disease prevalence has on the recruitment of new members, and $v$ and $w$ measure the influence of each infectious class on the recruitment function.

Treatment plays an important role in the establishment of public health policies. Its role - using model (1) - can be quantified through the basic reproductive number of the disease. Velasco-Hernández and Hsieh (1994) 
and Hsieh and Velasco-Hernández (1994) found that for model (1) with a constant recruitment rate $\Lambda$, the basic reproductive number $\mathcal{R}_{0}$ is given by

$$
\frac{c \beta_{0}}{\mu+\nu}+\frac{\sigma \mu / \Lambda}{\mu+\nu}\left(\frac{c^{\prime} \beta_{1}}{\mu+\nu^{\prime}}-1\right)
$$

We see that $\sigma=0$ gives the standard basic reproductive number for models without treatment. From (2) we observe the possibility of having a basic reproductive number greater than one even if $c \beta_{0}$ is sufficiently small, but the net transmission rate of treated individuals is not low enough. Of course, this is only possible if ill-designed or ineffective treatment programs romote rather than eradicate the spread of a fatal disease (Hsieh and VelascoHernandez, 1995; Anderson, Gupta and May, 1991).

In the next section we look at the combined effects of screening/treatment policies and variable recruitment in a model for the transmission dynamics of HIV.

\section{Model formulation}

In this section we follow the approach of Hadeler and Castillo-Chavez (1994) by explicitly introducing the non-core population as a reservoir for the sexually-active population consisting of the classes $S, U, I$ (the core group). We do not include the disease dynamics among non-core group members because our objective is to study the joint effects of treatment and prevalencedependent recruitment on HIV dynamics. We observe that more complex models, that is, with more compartments, have been studied in the past (metapopulations). However, such multicompartamental models are essentialy bilinear (mass-action law), and hence not comparable to those discussed in this article. In other words, our model is not a submodel of a general metapopulation model.

The paradigms that have been developed by Brauer et al. (1992), Hadeler and Castillo-Chavez (1994), and in this paper support the conclusion that the models that have been developed in the literature (see CastilloChavez 1989, Jewell et al. 1991, Sattenspiel and Castillo-Chavez 1990, May and Anderson 1989, Anderson and May 1991, and references therein) have only begun to scratch the surface of the possible realistic scenarios for the transmission dynamics of HIV. Heiderich et al. (1994) have modified related models to take into account the effects of delays in the behavioral response that governs the demographic interaction between the core and non-core 
groups and they have also found that delays can indeed destabilize simple epidemic models. Our extensions further confirm the high level of uncertainty due to behavioral changes in predicting disease dynamics are being carried out. The dynamics of disease transmission in a core group does not occur in a vacuum. The core-group is a part of a larger population. The non-core group provides new recruits and hence, the size of this pool is an important (scaling) factor (see Hadeler and Castillo-Chavez, 1994). Here, we study a homogeneous version of model (1) for which recruitment into the core population occurs at a rate $g(S, U, I)=r\left(\frac{v U+w I}{N}\right)$, for $v=w=1$ and $v=0, w=1$, that is, it depends on either the proportion of the core population infected or just the proportion of infected individuals under treatment. This function $r(x)$ is a decreasing function of $x$ on $0 \leq x \leq 1$ and $r(0)>0$. The recruitment function $r$ should strictly speaking depend on levels of prevalence of past times. Moreover, since recruits for the coregroup come from the non-core group, a maturation period has also to be considered. Heiderich et al (1994) have studied these effects on a simpler model. We do not consider such delays in this article.

To introduce our model we let $P, S, U$, and $I$ denote the sizes of the non-core, and the susceptible, untreated infectious, and treated infectious members of the core respectively. Following Hadeler and Castillo-Chavez (1994) we formulate the model as a general homogeneous system (Hadeler et al. 1988, Hadeler 1992, Busenberg and Hadeler 1990) and then specialize to the case of constant population size. The model equations are as follows:

$$
\begin{aligned}
\frac{d P}{d t} & =b(T-E)+\tilde{b} E-\operatorname{Pr}-\mu P, \\
\frac{d S}{d t} & =\operatorname{Pr}-B S-\mu S, \\
\frac{d U}{d t} & =B S-\sigma U-(\mu+\nu) U, \\
\frac{d I}{d t} & =\sigma U-\left(\mu+\nu^{\prime}\right) I,
\end{aligned}
$$

where $N=S+U+I, T$ is the total population, that is, $T=P+N$, $E=U+I$, and $b$ and $\tilde{b}$ are the birth rates of non-infected and infected groups respectively. The rate of acquisition of infection per susceptible individual $B$ is the same as in model (1).

We study the equilibria of (3) for the special case in which birth and death rates are identical for treated and untreated members given that total 
population size is constant. This requires the assumption $b=\tilde{b}$ and $\nu=$ $\nu^{\prime}=0$. The assumption $\nu=\nu^{\prime}=0$ while unrealistic allows us to carry out the analysis of a simplified model. Simulations for the general case confirm our results within a reasonable region of parameter space.

Under the assumption

$$
b=\tilde{b}, \quad \nu=\nu^{\prime}=0
$$

we have

$$
\frac{d T}{d t}=(b-\mu) T .
$$

If we assume $b=\mu$, then $T$ must be constant.

Now we rescale (3) under the above assumptions with the new nondimensional time $t^{\prime}=\mu t$. To limit the introduction of new parameters the new rescaled parameters in (3) are given the same names as before. Model (3) can be rewritten now with $\mu=1$. The per-susceptible incidence rate becomes

$$
\tilde{B}=c b_{0} \frac{U}{N}+c^{\prime} b_{1} \frac{I}{N}
$$

where

$$
b_{0}=\beta_{0} / \mu, \quad b_{1}=\beta_{1} / \mu .
$$

System (3) is therefore equivalent to $\left(I=\frac{d}{d t^{\prime}}\right)$ :

$$
\begin{aligned}
P^{\prime} & =T-P r-P \\
S^{\prime} & =\operatorname{Pr}-\tilde{B} S-S, \\
U^{\prime} & =\tilde{B} S-\sigma U-U, \\
I^{\prime} & =\sigma U-I .
\end{aligned}
$$

Normalizing the value of $N$, that is taking $N=1$ we conclude, without loss of generality, that the disease-free equilibrium is

$$
\left(P^{*}, S^{*}, U^{*}, I^{*}\right)=\left(\frac{1}{r_{0}}, 1,0,0\right)
$$

where $r_{0}=r(0)$. Equilibria of (4) with $E>0$ require $r(v U+v I)=1 / P$. If we let $\eta=c b_{0}+c^{\prime} b_{1} \sigma$, then the endemic equilibrium is given by

$$
P=\frac{1}{r}, \quad S=\frac{1+\sigma}{\eta}, \quad U=\frac{1}{1+\sigma}-\frac{1}{\eta}, \quad I=\frac{\sigma}{1+\sigma}-\frac{\sigma}{\eta}
$$


with

$$
E=U+I=1-\frac{1+\sigma}{\eta}
$$

We observe that $U>0$ and $E>0$ if and only if $\eta>1+\sigma$.

On average each untreated infective contributes $c b_{0}$ new infectives and each treated infective contributes $c^{\prime} b_{1}$ new infectives when introduced into a susceptible population with a mean infective period 1 . From $I=\sigma U$, the fraction of untreated infectives is $\frac{1}{\sigma+1}$ and the fraction of treated infectives is $\sigma /(\sigma+1)$. Hence, we heuristically arrive at the following expression for the basic reproductive number $\mathcal{R}_{0}$ :

$$
\mathcal{R}_{0}=\frac{c b_{0}}{1+\sigma}+\frac{\sigma c b_{1}^{\prime}}{1+\sigma}=\frac{\eta}{1+\sigma}
$$

and in terms of $\mathcal{R}_{0}$, we can write the endemic equilibrium population sizes as

$$
S=\frac{1}{\mathcal{R}_{0}}, \quad U=\left(\frac{\mathcal{R}_{0}-1}{\eta}\right), \quad I=1-S-U,
$$

provided that $\mathcal{R}_{0}>1$. In addition, since $\sigma>0$, we must have $\mathcal{R}_{0}<\eta$. Thus there is an endemic equilibrium provided

$$
1<\mathcal{R}_{0}<\eta .
$$

Now that we have established the region of parameter space in terms of $\mathcal{R}_{0}$ where the endemic equilibrium exists, we can proceed to study the local stability analysis of steady-state solutions of system (4).

\section{Effects of treatment on behavioral change}

The study of the effects of treatment on behavioral change is facilitated by the introduction of the re-scaled variables:

$$
p=\frac{P}{N}, \quad s=\frac{S}{N}, \quad u=\frac{U}{N}, \quad i=\frac{I}{N}, \quad e=u+i
$$

with $\mu=1$. Also we define

$$
\hat{B}=c b_{0} u+c^{\prime} b_{1} i
$$


In the re-scaled variables (4) becomes

$$
\begin{aligned}
p^{\prime} & =(1+p)[1-r(x) p] \\
u^{\prime} & =\hat{B}(1-e)-u(\sigma+p r(x)) \\
i^{\prime} & =\sigma u-i p r(x)
\end{aligned}
$$

where $x=i$ if $v=0, w=1$, or $x=u+i$ if $v=w=1$.

We first examine the special case in which treatment has no effect on the transmission rates of both infectious classes, that is, we assume that $c b_{0}=c^{\prime} b_{1}=\beta$. Since there is no distinction between treated and untreated infectious we take $v=1, w=1$, reducing model (7) to the following twodimensional system:

$$
\begin{aligned}
& p^{\prime}=(1+p)[1-r(e) p] \\
& e^{\prime}=\beta e(1-e)-\operatorname{epr}(e)
\end{aligned}
$$

where $e$ denotes the proportion of infected individuals (there is a single class of these because of our assumption $e$ ); $\beta$ denotes the effective contact rate rescaled by the length of the average sexually-active life of the population (and is therefore a non-dimensional quantity); while

$$
\eta=c b_{0}+c^{\prime} b_{1} \sigma=\beta(1+\sigma),
$$

and the basic reproductive reduces to $\beta$.

We proceed to study the stability properties of the equilibria of system (8) when $r=r(e)$, that is, when recruitment depends on the prevalence of the whole infected population. We treat the other case where $r=r(i)$ in the section 5 .

\subsection{Recruitment depending on the whole infected popula- tion}

Let $r=r(u+i)=r(e)$. It is easy to verify that the first quadrant of the phase plane is an invariant set and that every orbit of (8) is bounded.

The unique endemic equilibria of (8)is given by

$$
p^{*}=\frac{1}{r\left(e^{*}\right)}, \quad i^{*}=1-\frac{1}{\beta}
$$

provided that $\beta>1$. When $\beta<1$ there is only one equilibrium, the diseasefree equilibrium with $r_{0}=r(0)$. The equilibria for model (8) are identical to 
those found in the previous section with $\mathcal{R}_{0}=\beta$. Since the Jacobian matrix of $(8)$ is

$$
\left[\begin{array}{cc}
1-r(i)(1+2 p) & -(1+p) p r^{\prime}(e) \\
-r(e) e & \beta(1-2 e)-p\left(e r^{\prime}(e)+r(e)\right)
\end{array}\right]
$$

then the eigenvalues of this matrix at the disease-free equilibrium are $-1-r_{0}$, and $\beta-1$. Therefore, the disease-free equilibrium is locally asymptotically stable if and only if $\mathcal{R}_{0}<1$. Since there are no periodic orbits, the Poincaré Bendixson theorem shows that the asymptotic stability is global. If $\mathcal{R}_{0}>1$ the disease-free equilibrium is unstable (a saddle point) and there exists a unique endemic equilibrium.

A direct substitution into (9) of the values for the endemic equilibrium gives the following Jacobian matrix $J$ :

$$
J=\left[\begin{array}{cc}
-r^{*}-1 & -\frac{\rho}{r^{*}}\left(1+\frac{1}{r^{*}}\right) \\
-r^{*}\left(1-\frac{1}{\mathcal{R}_{0}}\right) & -\left(1+\frac{\rho}{\mathcal{R}_{0} r^{*}}\right)\left(\mathcal{R}_{0}-1\right)
\end{array}\right]
$$

where $\rho=\frac{\partial r}{\partial i}$ is evaluated at $e^{*}, \mathcal{R}_{0}=\beta$, and in this simplified case $r^{*}=$ $r\left(e^{*}\right)$. By hypothesis, $\rho<0$. and therefore, the stability of $J$ is determined by the roots of the characteristic polynomial of $J$

$$
\lambda^{2}+a_{0} \lambda+a_{1}=0
$$

where

$$
\begin{aligned}
& a_{0}=r^{*}+1+\frac{R_{0}-1}{R_{0}}\left(\mathcal{R}_{0}+\frac{\rho}{r^{*}}\right) \\
& a_{1}=\left(r^{*}+1\right)\left(\mathcal{R}_{0}-1\right)>0 .
\end{aligned}
$$

The endemic equilibrium is asymptotically stable if and only if $a_{0}>0$ that is, if and only if

$$
\mathcal{R}_{0}\left(r^{*}+1\right)>-\left(\mathcal{R}_{0}-1\right)\left[\mathcal{R}_{0}+\frac{\rho}{r^{*}}\right]
$$

This quadratic inequality is satisfied if $\mathcal{R}_{0}$ is between the roots of the corresponding quadratic equation. These roots lie in the interval $\left(1,-\frac{\rho}{r^{*}}\right)$ provided $|\rho| / r^{*}>1$.

It follows that for $\mathcal{R}_{0}>-\rho / r^{*}$, the endemic equilibrium is asymptotically stable. However, for $\mathcal{R}_{0}<-\rho / r^{*}$, there exists numbers $1<\omega_{0}<\omega_{1}<$ 
$-\rho / r^{*}$ such that for $\mathcal{R}_{0} \in\left(\omega_{0}, \omega_{1}\right)$ the endemic equilibrium is unstable. From (8) one can also see that the set

$$
D=\left\{(p, e): \quad 0<p \leq \frac{1}{r^{*}}, \quad 0<e \leq 1\right\}
$$

is bounded and invariant under the flow generated by (8). Therefore, the Poincaré-Bendixon theorem implies the existence of at least a periodic orbit within $D$ which is the $\omega$-limit set of orbits in $D$. Thus, the following result has been established:

Theorem 1 Suppose $\rho<0$. Then, whenever $\mathcal{R}_{0}<1$ the unique equilibrium point

$$
p^{*}=\frac{1}{r_{0}}, \quad e^{*}=0
$$

of (8) is globally asymptotically stable.

If $\mathcal{R}_{0}>1$ there exists a second equilibrium,

$$
p^{*}=\frac{1}{r\left(e^{*}\right)}, \quad e^{*}=1-\frac{1}{\mathcal{R}_{0}},
$$

with the following properties. For any initial conditions $\left(p_{0}, e_{0}\right)$ in $D$ and provided $|\rho| / r^{*}>1$, if $1<\mathcal{R}_{0} \in\left(\omega_{0}, \omega_{1}\right)$ then the equilibrium is unstable and there exists an attracting periodic orbit which is the $\omega$ limit set of orbits in $D$. Otherwise if $1<\mathcal{R}_{0}<\omega_{0}$ or $\omega_{1}<\mathcal{R}_{0}<\infty$, the equilibrium is globally asymptotically stable in $D$.

In Figure 1 we show the parameter plane $\left(-\rho / r^{*}, \mathcal{R}_{0}\right)$ for the particular recruitment function $r(e)=\exp (-\kappa e)$. The graph shows where Hopf bifurcations occur. It divides the parameter space into two regions. We see that bifurcations may occur for values of $\mathcal{R}_{0}$ just above 1 for a wide range of $|\rho|$ values. The region to the left of the graph corresponds to asymptotic stability of the endemic equilibrium, and the area on the right corresponds to limit cycle solutions around this point. We state then the following conjecture that is verified for numerical simulations:

Conjecture 1 Refer to Figure 1; let $|\rho| / r^{*}>1$. Then, for any initial condition $\left(p_{0}, e_{0}\right)$ in the set described in (10), the endemic state $\left(p^{*}, e^{*}\right)$ is a globally asymptotically stable equilibrium (stable spiral) in Region $I$ and an unstable focus associated with a globally stable limit cycle in Region II. 
If we reinterpret $|\rho|$ as an index of risk perception (the greater the value of $|\rho|$ the faster the decay of the recruitment rate), the lower the risk index, the larger the non-core population at equilibrium needed to give rise to periodic behavior.

\subsection{The role of treatment}

A successful treatment program is characterized here as a program satisfying the inequality $c b_{0}>c^{\prime} b_{1}$. Furthermore, it is assumed here that the perception of risk by an individual that wants to join the core group is also function of the number of infected individuals under treatment. Two cases are studied in this section: one considers the situation where the recruitment rate into a core-group is negligible compared with the rates of infection and treatment dynamics. The second deals with a scenario where the treatment program is suspended.

Assume that $v=0, w=1$, and $\sigma>0$. System (7) has a disease-free equilibrium with $u=0, i=0$ and $p=1 / r(0)$ and, provided $\mathcal{R}_{0}>1$, it also has an endemic equilibrium $\left(p^{*}, u^{*}, i^{*}\right)$ given by

$$
\begin{aligned}
p^{*} & =1 / r^{*}, \\
u^{*} & =\frac{1}{\sigma+1}-\frac{1}{\eta}=\frac{\left(\mathcal{R}_{0}-1\right)}{(\sigma+1) \mathcal{R}_{0}}, \\
i^{*} & =\sigma u^{*},
\end{aligned}
$$

where $e^{*}=u^{*}+i^{*}, r^{*}=r\left(i^{*}\right)$ and, as before, $\eta=c b_{0}+c^{\prime} b_{1}, \mathcal{R}_{0}=\frac{\eta}{\sigma+1}$. The Jacobian matrix $J$ of (7) at an equilibrium is

$$
\left[\begin{array}{ccc}
1-p r-(1+p) r & 0 & -p(1+p) r^{\prime} \\
-u r & -(\sigma+\tilde{B})-p r+c b_{0} s & -\tilde{B}-p u r^{\prime}+c^{\prime} b_{1} s \\
-i r(i) & \sigma & -p\left(r+i r^{\prime}\right)
\end{array}\right]
$$

where $1=s+e$, and $\frac{d r}{d i}=r^{\prime}(i)<0$ for all $i$.

At the disease-free equilibrium $\left(\frac{1}{r(0)}, 0,0\right)$, the matrix is

$$
J=\left[\begin{array}{ccc}
-r_{0}-1 & 0 & -\frac{r^{\prime}(0)}{r_{0}}\left(1+\frac{1}{r_{0}}\right) \\
0 & c b_{0}-\sigma-1 & c^{\prime} b_{1} \\
0 & \sigma & -1
\end{array}\right]
$$

If $\mathcal{R}_{0}<1$, that is, if $\eta<\sigma+1$, it is not difficult to verify via the RouthHurwitz criteria that the eigenvalues of $J$ have negative real parts and thus the disease-free equilibrium is asymptotically stable. 


\subsubsection{Quasi-steady state of the non-core/core proportion}

When the relative sizes of the non-core/core populations remain relatively constant, we can approximate our original three-dimensional model (eq. 7) with a two-dimensional system. We assume that the growth of $p=P / N$ is slow when compared with the disease process in the core group. Under this assumption we set $p^{\prime}=0$. Solve this equation for $p$ and substitute the value of $p$ into the other two equations to obtain:

$$
\begin{aligned}
u^{\prime} & =\hat{B}(1-e)-u(\sigma+1), \\
i^{\prime} & =\sigma u-i .
\end{aligned}
$$

Local stability properties of the disease-free equilibrium $(u, i)=(0,0)$ of this model are identical to the ones derived from (11). However, at the endemic equilibrium we have the Jacobian matrix

$$
J_{s s}=\left[\begin{array}{cc}
\frac{c b_{0}}{\mathcal{R}_{0}}+1-\left(1+c b_{0}+\sigma\right) & \left.1-\mathcal{R}_{0}+\frac{c^{\prime} b_{1}}{\mathcal{R}_{0}}\right) \\
\sigma & -1
\end{array}\right] .
$$

Thus we have

$$
\operatorname{Trace}\left(J_{s s}\right)=\frac{c b_{0}}{\mathcal{R}_{0}}-\left(1+\sigma+c b_{0}\right), \operatorname{Det} J_{s s}=c b_{0}-(1+\sigma)+\mathcal{R}_{0} \sigma .
$$

Under the assumption that $\mathcal{R}_{0}>1$, we see that the Routh-Hurwitz criterion implies that the endemic equilibrium is always locally asymptotically stable. In fact, it is straightfroward to check that it is globally asymptotically stable in $\Omega$.

\subsubsection{Suspension of treatment}

Suppose now that we begin with a non-zero proportion of treated infectious individuals and cease treatment (making $\sigma=0$ ). The model becomes

$$
\begin{aligned}
p^{\prime} & =(1+p)[1-r(i) p] \\
u^{\prime} & =\hat{B}(1-e)-\operatorname{upr}(i) \\
i^{\prime} & =-i p r(i)
\end{aligned}
$$

Now $\eta=c b_{0}, \mathcal{R}_{0}=\eta$, and the endemic equilibrium is given by

$$
p^{*}=1 / r^{*}, \quad e^{*}=1-\frac{1}{\eta}, \quad u^{*}=\epsilon^{*}, \quad i^{*}=0
$$


Since $\sigma=i^{*}=0$, the eigenvalues are -1 and the eigenvalues of

$$
\left[\begin{array}{cc}
-\left(1+p^{*}\right) r & 0 \\
-u r & -\eta+1
\end{array}\right]
$$

Therefore, the endemic equilibrium is always asymptotically stable when it exists.

\section{Conclusions}

We have considered in this paper the effect of treatment on the dynamics of a highly active group of homosexual individuals. We considered two situar tions. First we considered the case where treatment, represented by $\sigma$ in our model, affects only the contact rates of infected persons (treated infectious individuals are less infectious than untreated). In this scenario, treatment does not affect directly the recrutiment rate since recruitment depends on the total infected proportion (we take $v=w=1$ ). This situation exemplifies the situation where the perception of risk is assessed from information on the total prevalence in the core group $(u+i)$. The second situation assumes that treatment affects both the infectivity of treated individuals (which goes down) and the recruitment rate $(v=0, w=1)$. Therefore, recruitment depends only on the proportion of infected individuals. Our results indicate that periodic behavior is possible in the dynamics of (7). We have explored different cases of equation (7) where periodic and non-periodic behavior is present.

The following result states the conditions under which no periodic behavior is possible for the case $v=0, w=1(r=r(i))$ :

Theorem 2 When either $p^{\prime}=0$ as in equqtion (12), or $\sigma=0$, as in equation (14), the endemic state exists and it is globally asymptotically stable whenever $\mathcal{R}_{0}>1$.

Note that the limiting system that results by taking $t \rightarrow \infty$ in (7), in the case $v=0, w=1$, is

$$
\begin{aligned}
& p^{\prime}=(1+p)[1-r(0) p] \\
& u^{\prime}=c b_{0} u(1-u)-u p r(0)
\end{aligned}
$$

since in the absence of treatment $i \rightarrow 0$. This equation is exactly (8) for $r=$ constant. It cannot present periodic behavior, as can be seen from 
(9) and the discusion therein. Obviously, (8) exhibits richer behavior than either of the two special cases just described.

Theorem 2 deals with a two-dimensional approximations to (7). It remains to find out if periodic behavior is possible in the general case-a three-dimensional model. We have numerically explored its asymptotic behavior for various values of the treatment parameter $\sigma>0$ and for the particular recruitment function $r(v u+w i)=\exp (-\kappa(v u+w i))$, where $\kappa$ can be interpreted as an index of risk perception.

For the numerical simulations, we considered a whole range of cases by taking $w=1$ and $0 \leq v \leq 1$. Figures $2 \mathrm{a}$ and $2 \mathrm{~b}$ show the results of numerical simulations for the case $v=0, w=1\left(c b_{0}=30\right.$ and $c^{\prime} b_{1}=$ 20 in this case). These values correspond to a contact rate of $c \beta_{0}=1$, $c^{\prime} \beta_{1}=0.666$, and $\mu=1 / 30$. Figures $3 \mathrm{a}$ and $3 \mathrm{~b}$ show the time evolution of the cases illustrated in the previous figures but with a higher $\kappa$. For the given values of the contact rates, periodic behavior develops when $\kappa$ increases from 4 to 6 through a Hopf bifurcation. The treatment rate $\sigma>0$ induces a positive flow of untreated individuals into the treatment class. As the prevalence of this compartment rises, the magnitude of the recruitment function quickly decreases with the consequent decrease in the size of the core population (see Figure 3). When this occurs the proportion of treated individuals crashes and the size of the core group increases mainly by the increase of new recruits that go into the untreated compartment. As these are treated, the perception of risk increases too and the process is repeated once more. The same result follows if in equation (3) $\sigma$ constant is replaced by $\sigma T / N$. This form of treatment rate is closer to the original definition given in equation (1). Equation (7) is now the same except that $\sigma$ must be replaced by $\sigma /(1+p)$.

Figure 4 shows the asymptotic dynamics of (7) when the proportion of untreated infected persons plays an increasing role in the recruitment of new core group members. As $v$ increases from 0 to 1 , the fluctuations in the proportion of untreated infecteds increase too, until the equilibrium point becomes asymptotically stable. When $v=1, w=1$, the equilibrium point is asymptotically stable for, at least, $\kappa<13$. From the point of view of the objective of this work, it is more relevant to explore the changes in asymptotic behavior of (7) when the contact rates $c \beta_{0}$ and $c^{\prime} \beta_{1}$ vary. Figure 5 shows the behavior of the system when $v=w=1$. In this case, equation (7) turns out to be more sensitive to the contact rates $c \beta_{0}$ and $c^{\prime} \beta_{1}$. Figure 7 shows the results of taking $c \beta_{0}=3.0$ and varying $c^{\prime} \beta_{1}$ from 0.2 to 1 (equivalently, varying $c^{\prime} b_{1}$ from 6 to 1 ). Essentially, when $c \beta_{1}$ decreases, the 
equilibrium point becomes asymptotically stable. It follows that, for a given level of treatment and risk index, higher levels of infectivity are necessary to produce periodic behavior. In other words, less efficient treatment can produce oscillations in (7).

Simple models that take into account disease-level effects on the recruitment rate of susceptible members into the core population as well as the effects of treatment rates on disease dynamics exhibit more complex dynamics than those that exclude them (but see Heiderich et al. 1994). It is important to try to sort out the time scales at which behavioral changes and treatment effects begin to impact disease dynamics and public policy.

Disease-prevalence recruitment rates can trigger the appearance of periodic solutions through Hopf bifurcations as we vary the treatment rate. Models that explicitly exclude the time evolution of the non-core population (which implicitly provides new recruits into the core group) may or may not be capable of exhibiting periodic outbreaks as we vary the treatment rate. Our numerical simulations suggest that sustained periodic behavior is in fact possible in the absence of treatment. However, when we take into account the population dynamics of the non-core populations then treatment rate plays an important role on the resulting (qualitative) disease dynamics. Variations on the treatment rate may lead to drastic changes in the transient behavior of solutions as $\mathcal{R}_{0}$ depends on the treatment rate. In fact, the three dimensional models can undergo bifurcations of the endemic equilibrium as $\mathcal{R}_{0}$ increases from 1 . Similar phenomena have been explored by Hadeler and Castillo-Chavez (1994) and Feng and Thieme (1995).

The results presented in this paper are an attempt to explore the dynamics of infectious diseases in non-isolated core groups. We have emphasized the qualitative properties of models that incorporate into their framework state-dependent recruitment and core-specific treatment rates. We hope that their study will add to our understanding of the dynamics of diseases such as HIV/AIDS that exist and evolve in complex social environments.

Acknowledgements This research was partially supported by NSF grant DEB-925370 (Presidential Faculty Fellowship Award) to Carlos CastilloChavez and by the U.S. Army Research Office through the Mathematical Science Institute of Cornell University (contract DAAL03-91-C-0027). Jorge X. Velasco-Hernández' research was partially supported by the Consejo Nacional de Ciencia y Tecnología de México (CONACYT) through grant 400200-5-3551E. 


\section{References}

- Anderson, R.M., Gupta, S. and R.M. May (1991) Potential of communitywide chemotherapy or immunotherapy to control the spread of HIV-1. Nature350: 365-369.

- Anderson, R.M., and R.M. May (1991). Infectious diseases of humans. Oxford Science Publications. Great Britain.

- Baldwin J.D., Baldwin, J.I. (1988) Factors affecting AIDS-related sexual risk-taking behavior among college students. J. Sex Research 25, 181-196.

- Blythe, S. P., Brauer, F., Castillo-Chavez, C., Velasco-Hernández, J.X. (1992a) Sexually-transmitted diseases with recruitment. Biometrics Unit Technical Report BU-1193-M), Cornell University.

- Blythe, S.P., Brauer, F. and Castillo-Chavez, C. (1992b) Demographic recruitment in sexually transmitted disease models. Biometrics Unit Technical Report BU-1154-M, Cornell University.

- Brauer, F. C.Castillo-Chavez and J.X. Velasco-Hernández. (1993) Recruitment effects in heterosexually transmitted diseases. Technical Report. Mathematical Sciences Institute. Cornell University.

- Busenberg, S., Hadeler, K.P.,(1990) Demography and epidemics. Math. Biosc. 101, 63-74.

- Castillo-Chavez, C. (ed.) (1989). Mathematical and statistical approaches to AIDS epidemiology. Lecture Notes in Biomathematics, Vol. 83, Springer-Verlag: Berlin.

- [15] Castillo-Chavez, C. and H.R. Thieme (1993). Asymptotically autonomous epidemic models. In O. Arino, M. Kimmel (eds.) Proc. Third Intern. Conf. on Mathematical Population Dynamics (to appear)

- Curran, J. W., Jaffe, H.W., Hardy, A.M., Morgan, M.W., Selik, R.M., Dondero, T.J. (1988) Epidemiology of infection and AIDS in the United States. Science 293, 610-616. 
- Evans, B. A., McLean, K.A., Dawson, S.G. et al. (1989) Trends in sexual behavior and risk factors for the HIV infection among homosexual men, 1984-1987. Brit. Med. J. 298, 215-218.

- Fineberg, H. V. (1988) Education to prevent ADS: prospects and obstacles. Science 239, 592-596.

- Hadeler, K.P. (1992) Periodic solutions of homogeneous equations. J. Diff. Equ. 95, 183-202.

- Hadeler, K. and C. Castillo-Chavez (1994) A core group model for disease transmission. Preprint.

- Hadeler, K.P., Müller, J. (1993) Vaccination stategies for sexually transmitted diseases. In: Arino, O., Axelrod, D., Kimmel, M. (eds.) Proc. Conf. Population Dynamics, Pau (France) 1992.

- Hadeler, K.P., Waldstätter, R., Wörz-Busekros, A. (1988) Models for pair formation in bisexual populations. J. Math. Biol. 26, 635-649.

- Heiderich, K., Huang W., and Castillo-Chavez C. (1994). Nonlocal response in a simple epidemiological model. Preprint

- Hethcote, H. W., Yorke, J.A. (1984) Gonorrhea: Transmission Dynamics and Control. Lecture Notes in Biomathematics 56, SpringerVerlag.

- Hsieh, Y-H., and J.X. Velasco-Hernández (1995) Community-wide treatment and HIV. Biosystems, to appear.

- Jewell, N.P., K. Dietz, and V.T. Farewell (1991). AIDS epidemiology: Methodological issues. Birkhäuser: Boston.

- Martin, J. L. (1987) The impact of AIDS in gay male sexual behavior patterns in New York City. Am. J. of Pub. Health 77 578-581.

- May, R.M. and R.M. Anderson. (1989). The transmission dynamics of human immunodeficiency virus (HIV). Phil. Trans. R. Soc. London, B, 321, 565-607.

- McKusick, L., Wiley, J.A., Coates, T.J., Stall, R., Saika, B., Morin, S., Hortsman, C.K., Conant, M.A. (1985) Reported changes in the sexual behavior of men at risk for AIDS, San Francisco, 1983-1984: the AIDS behavioral research project. Public Health Reports 100, 622-629. 
- Saltzman, S.P., Stoddard, A.M., McCusker, J., Moon, M.W., Mayer, K.H. (1987) Reliability of self-reported sexual ehavior risk factors for HIV in homosexual men. Public Health Report 102, 692- 697.

- Sattenspiel, L. and C. Castillo-Chavez. (1990). Environmental context, social interactions, and the spread of HIV. American Journal of Human Biology, 2, 397-417.

- Shechter, M.T., Craib, K.J.P., Willoughby, B., Douglas B., Alastair McLeod, W., Maynard, M., Constance, P., and O'Shaughnessy, M. (1988) Patterns of sexual behavior and condom use in a cohort of homosexual men. Amer. J. Publ. Health 78, 1535-1538.

- Shilts, R. (1987) And the band played on. San Martin's press, New York.

- Thieme, H.R. (1992): Convergence results and a Poincaré-Bendixson tricho-tomy for asymptotically autonomous differential equations. $J$. Math. Biol. 30, 755-763

- Thieme, H.R. (1993a): Asymptotically autonomous differential equations in the plane, Rocky Mt. J. Math. (to appear)

- Thieme, H.R, (1993b), Asymptotically autonomous differential equar tions in the plane II. Stricter Poincaré-Bendixson type results, Diff. Integral Eq. (to appear)

- van Griensven, G.J.P., de Vroome, E.M.M, Goudsmit, J., Coutinho, R.A. (1989a) Changes in sexual behavior and the fall in incidence of HIV infectin among homosexual men. Br. Med.J. 298, 218-221.

- van Griensven, G.J.P, de Vroome, E.M.M., Tielman, R.A.P., Goudsmit J. A. A. P., de Wolf F., van der Noordaa J., and Coutinho R. A. (1989b) Effect of human immuno-deficiency virus (HIV) antibody knowledge on high-risk sexual behavior with steady and non-steady partners among homosexual men. Am.J. Epidem. 129, 596-603.

- Velasco-Hernández, J.X. and Y-H. Hsieh (1994) Modeling the effect of treatment and behavioral change in HIV-transmission dynamics. $J$. Math. Biology 32:233-249 
- Wiktor, S.Z., Biggar, R.J., Melby, M., Ebbesen, P., Colclough, G., Di Gioia, R., Sanchez, W.C., Grossman, R.J., Goedert, J. (1990) Effects of knowledge of human immunodeficiency virus infection status on sexual activity among homosexual men. J.of AIDS 3, 62-68.

- Wilkenstein, W. Jr., Wiley, J.A., Padian, N.S., Samuel, M., Shiboski, S., Ascher, M. S., and Levy J. A. (1988) The San Francisco Men's Health Study, continue decline in HIV seroconversion rates among homosexual/bisexual men. Am. J. Public Health 78, 1472-1474. 


\section{Figure captions}

- Figure 1 Parameter plane $\left(|\rho| / r^{*}, \mathcal{R}_{0}\right)$ for equation (8). The graph shows the line along which Hopf bifurcations occur. As $\mathcal{R}_{0}=\beta$ increases from 1 , the nontrivial endemic equilibrium loses asymptotic stability, giving rise to periodic behavior. For higher values of $\mathcal{R}_{0}$, asymptotic stability is regained.

- Figure 2 Numerical computations for model (7). Parameter values are $c b_{0}=30, c^{\prime} b_{1}=20, \sigma=1.5$, and $v=0, w=1$. (a) $\kappa=4$. This graph illustrates the case where recruitment depends only on the prevalence of treated infected individuals. The endemic equilibrium point is asymptotically stable; (b) same as in a) but with $\kappa=6$. The asymptotic stability of the endemic equilibrium has given rise to periodic solutions.

- Figure 3 Time-evolution plot of solutions to (7) for the parameter values $c b_{0}=30, c^{\prime} b_{1}=20, \sigma=1.5$, and $v=0, w=1$ and $\kappa=9$. Simulations run for 10 generations (approximately 300 years). Note that the period of the solution is about 80 years. (a) Graph of the proportions of untreated and treated proportions. (b) Graph of the non core/ core population ratio.

- Figure 4 Numerical computations for model (7). Parameter values are $c b_{0}=30, c^{\prime} b_{1}=20, \sigma=1.5$, and $\kappa=9$. This graph illustrates the different solutions of our model when $v$ increases from $0(r=r(i))$ to $0.5(r=r(0.5 u+i))$. Note that limit cycles disappear. as $v$ increases.

- Figure 5 Numerical computations for model (7). Parameter values are $c b_{0}=10, \sigma=1.5$, and $\kappa=9$. This graph illustrates the different solutions of our model when $c^{\prime} b_{1}$ increases from 1 to 6 . Note that limit cycles disappear as $c^{\prime} b_{1}$ increases. 


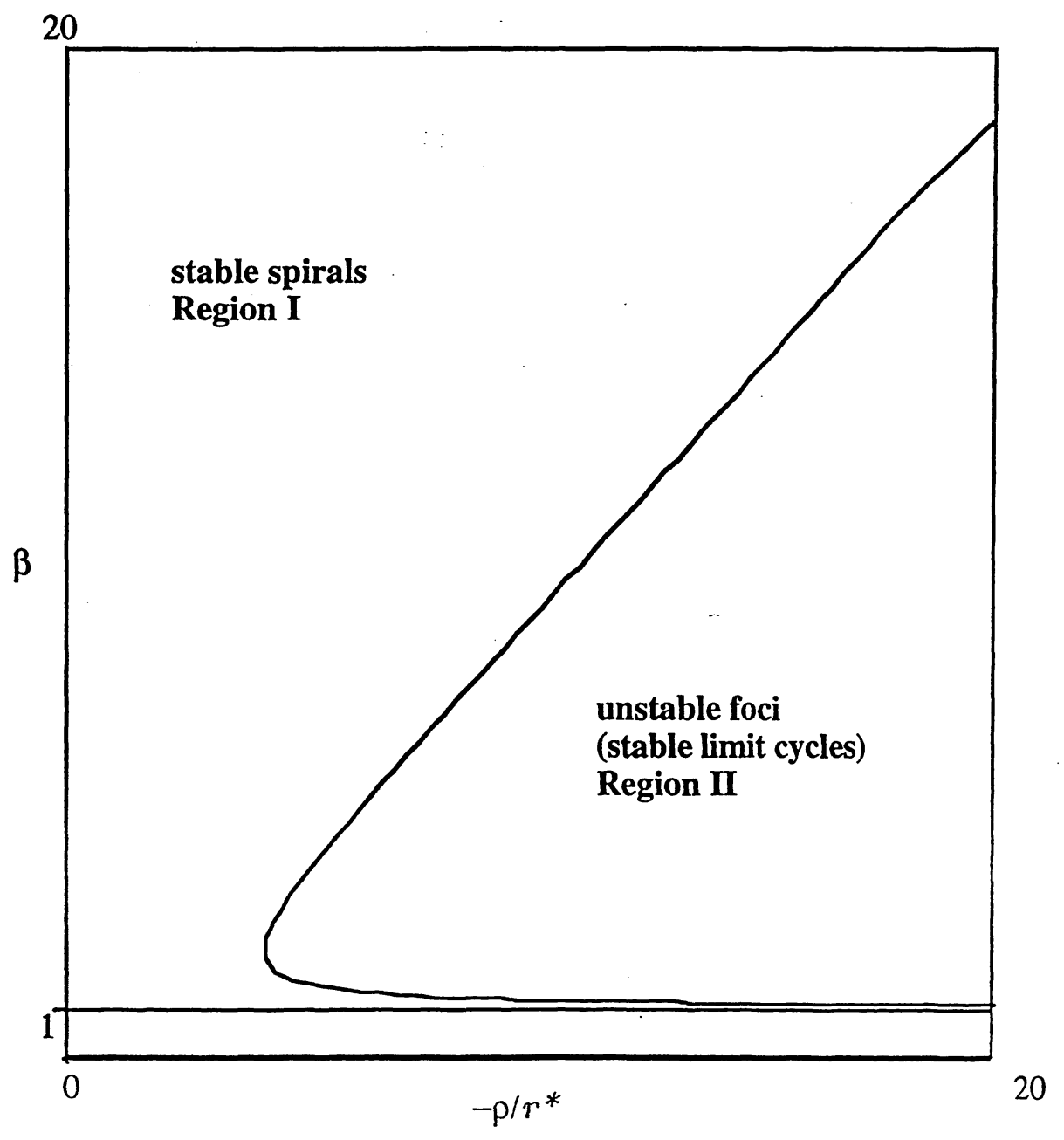




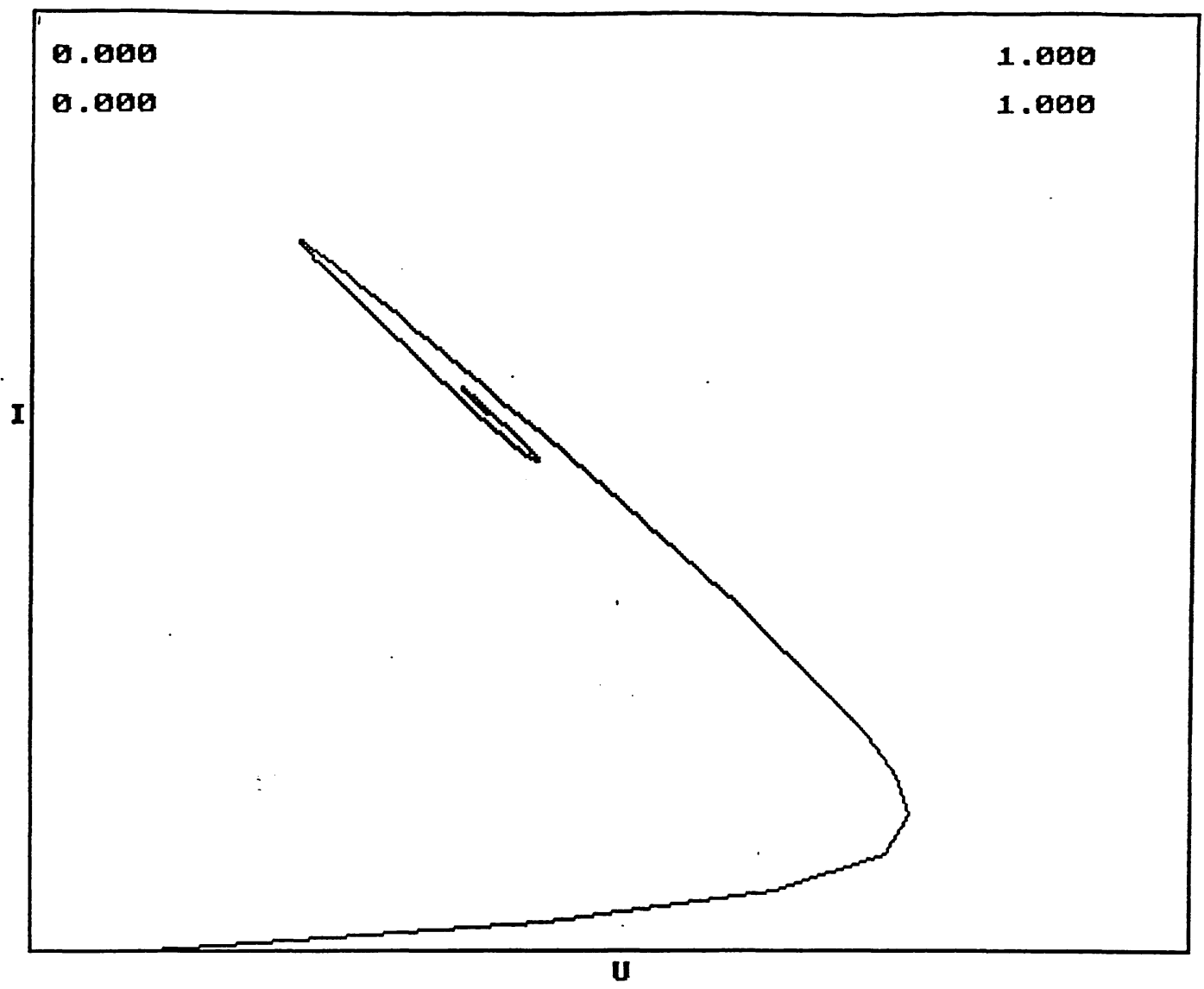

$2 a$ 


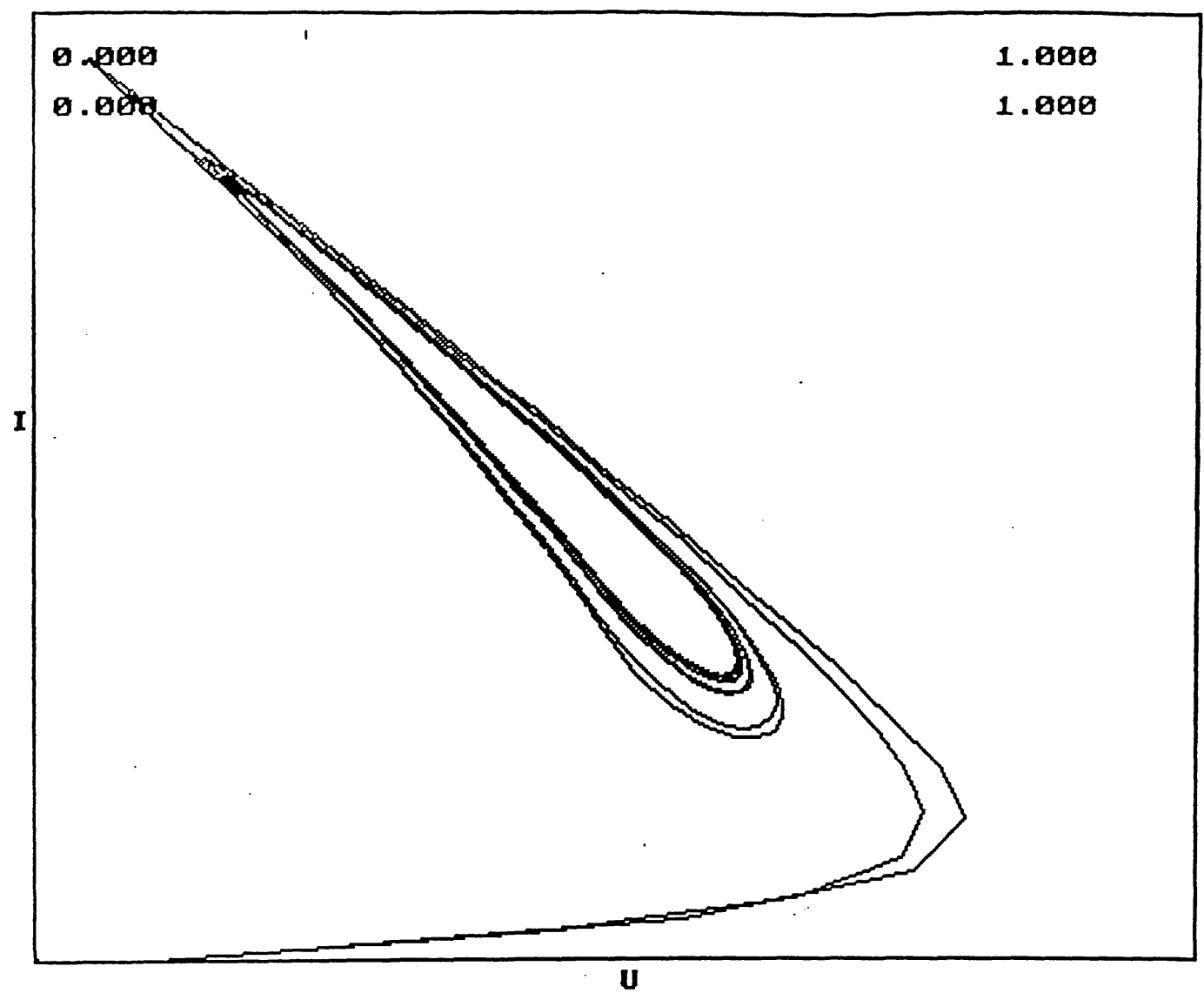

$2 b$ 


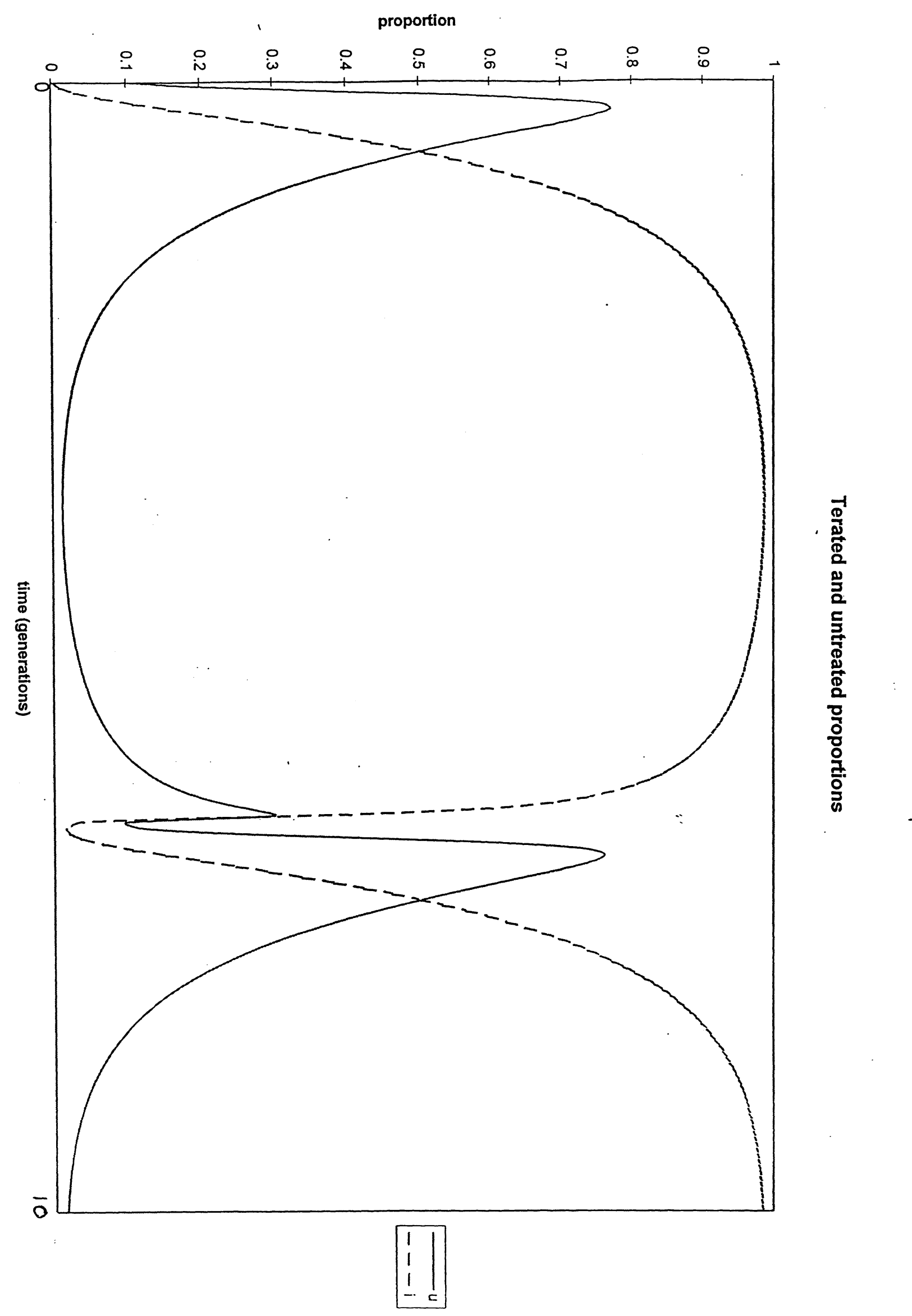

$3 a$ 


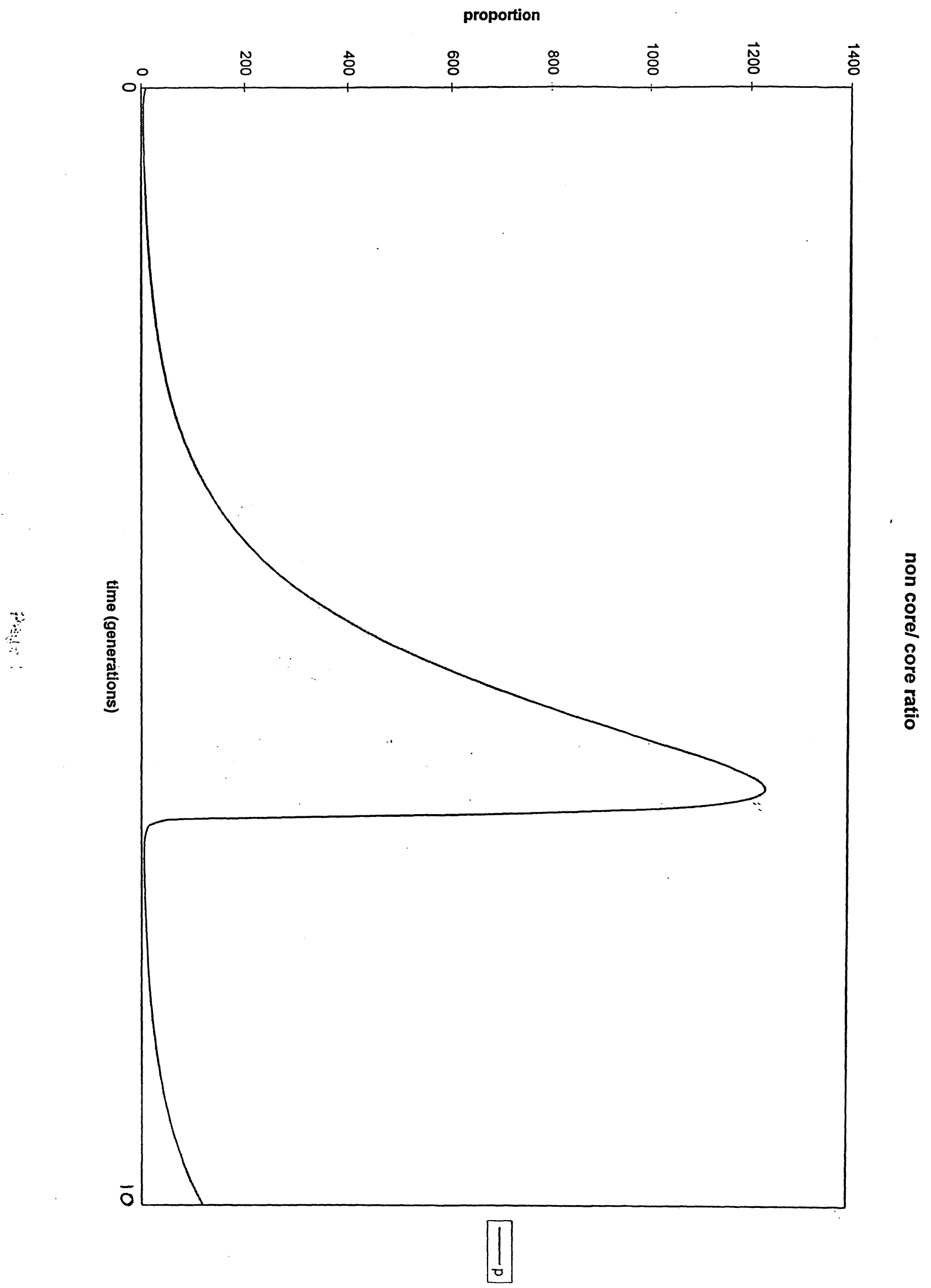




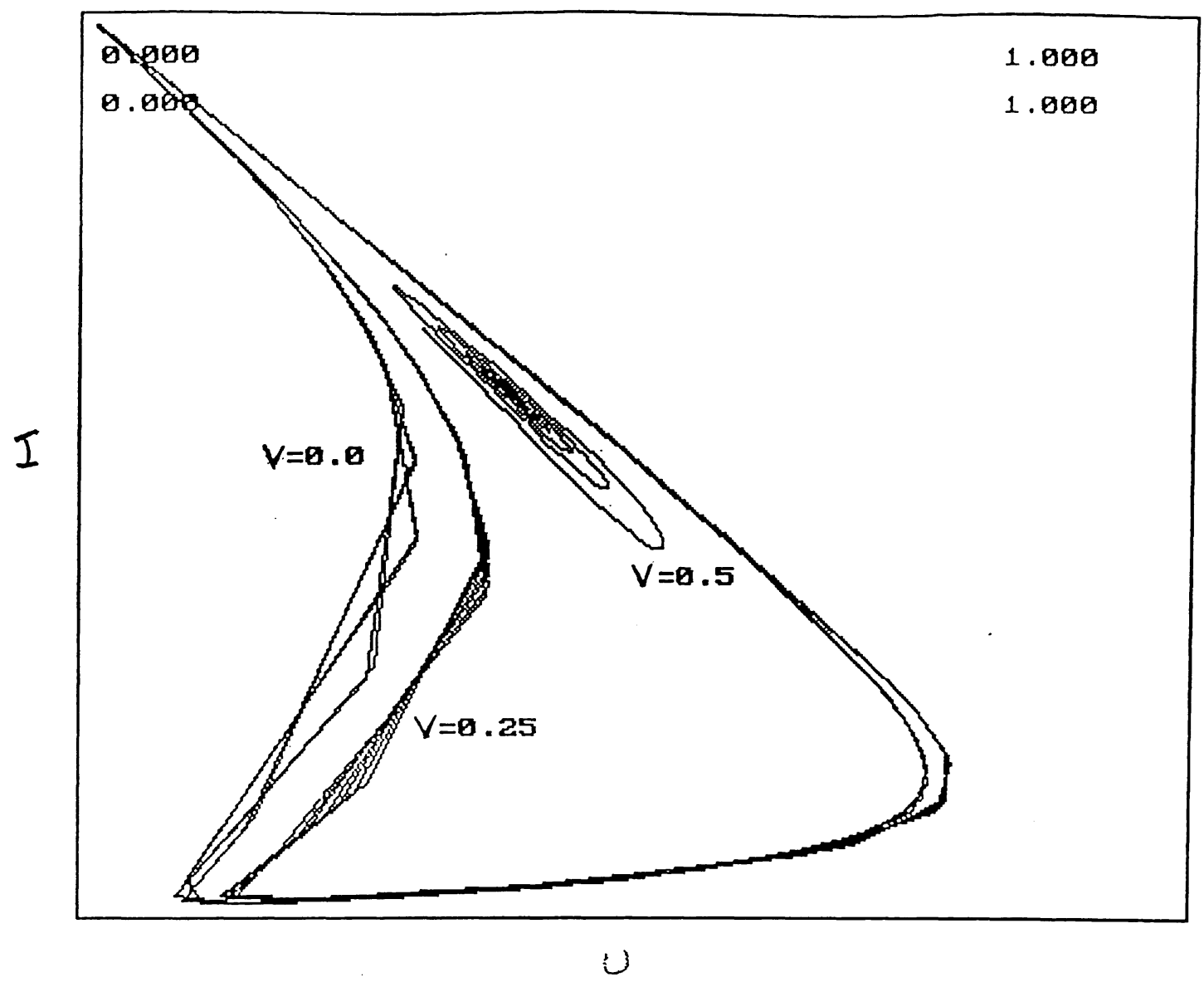

4 


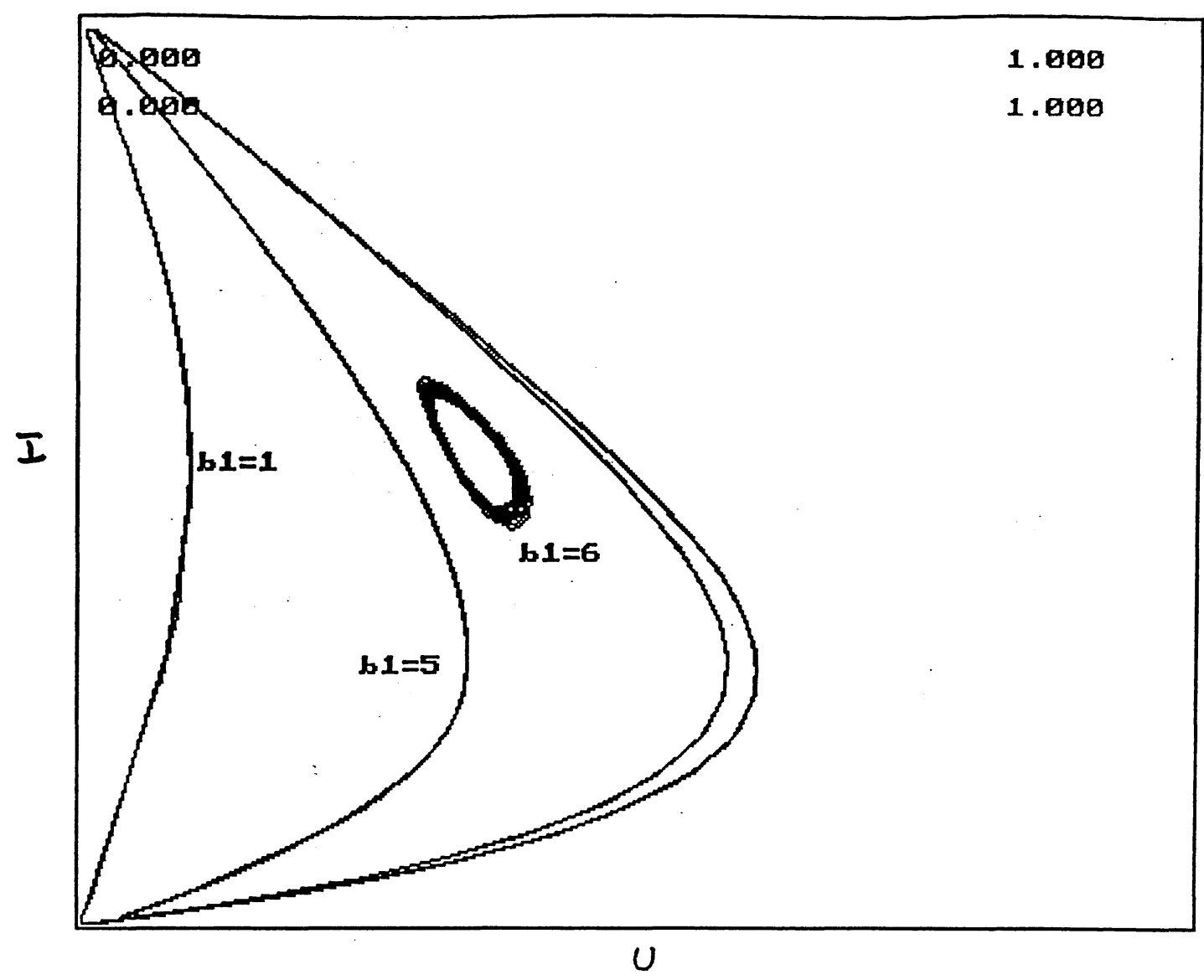

